Classification

Physics Abstracts

$42.60 \mathrm{~K}-42.78-44.10$

\title{
Effets thermiques induits par des dispositifs optiques d'uniformisation de faisceau laser
}

\author{
Li Junchang $\left(^{*}\right)$ et J. Merlin \\ INSA Lyon, Bât. 502, G.E.M.P.P.M. (U.A. 341) Calfetmat., 69621 Villeurbanne, France
}

(Reçu le 2 avril 1990, révisé le 6 septembre 1990 et le 25 octobre 1990, accepté le 9 novembre 1990)

\begin{abstract}
Résumé. - Afin d'améliorer la qualité du traitement superficiel des matériaux avec un faisceau laser de puissance", nous avons étudié plusieurs dispositifs susceptibles de modifier la répartition d'énergie du faisceau primaire. Le présent travail montre théoriquement l'intérêt des dispositifs optiques de recombinaison de faisceau vis-à-vis des effets thermiques induits. Les analyses réalisées s'appuient sur l'étude comparative des répartitions de température obtenues en utilisant soit un faisceau primaire gaussien soit un faisceau transformé par un de nos dispositifs dans le cas de matériaux de différentes natures considérés comme semi-infinis, homogènes et avec des caractéristiques thermiques constantes. Cette étude permet de constater que l'influence des interférences résultant de la superposition des sous-faisceaux cohérents est tout à fait négligeable dans la plupart des situations rencontrées dans la pratique, mais par contre que les phénomènes de diffraction doivent être pris en compte dans un certain nombre de cas. Enfin on souligne le fait qu'il n'existe pas une répartition idéale applicable à toutes les situations mais des répartitions optimales à définir selon les conditions de chaque traitement.
\end{abstract}

\begin{abstract}
In order to increase the quality of superficial heat treatment of materials with high power laser beam, several devices being able to modify the initial energy beam repartition have been studied. The present work shows the theoretical interest of these devices in relation to the induced thermal effects. Comparison of thermal profiles have been performed between a primary Gaussian beam and transformed beam for different materials which were considered like homogeneous and semi-infinite solids with constant thermal properties. This study shows that the interferences due to the superposition of coherent beams have negligeable effects but that the diffraction phenomena must be taken into account in most practical situations. At last it appears that one ideal repartition does not exist whatever may be the operating condition but optimal repartition must be defined for each situation.
\end{abstract}

\section{Introduction.}

Dans le but de traiter superficiellement de manière homogène des matériaux avec un faisceau laser de puissance nous avons étudié plusieurs dispositifs susceptibles de modifier la répartition d'énergie du faisceau primaire [1-3]. Les études théoriques et expérimentales ont

$\left(^{*}\right)$ Adresse permanente: Groupe d'Etudes Appliquées au Laser, Institut de Technologie de Kunming, Chine. 
montré [4] qu'après la transformation par un de ces dispositifs, un faisceau gaussien (TEM $\mathrm{T}_{00}$ ) pouvait être transformé en une tache carrée ou en une «bande gaussienne » présentant plus ou moins d'anomalies. Cependant, l'interaction laser-matériau est un processus physique complexe, et le champ de température superficiel dépend non seulement de la répartition d'énergie du faisceau incident mais encore d'autres paramètres tels que le temps d'interaction (ou la vitesse de balayage), les paramètres thermiques du matériau, ou encore les inhomogénéités de la matière. Par conséquent, un éclairement homogène du laser ne suffit généralement pas pour conduire à une répartition de température homogène dans la zone traitée et inversement, des perturbations dans la répartition d'énergie incidente peuvent avoir des conséquences plus ou moins grandes vis-à-vis des effets thermiques. Pour être sûr de l'intérêt de nos dispositifs vis-à-vis de l'utilisation pour le traitement superficiel des matériaux, l'étude théorique des effets thermiques induits par ces dispositifs est donc nécessaire, d'autant que toutes nos études sont basées sur la décomposition du faisceau primaire en sous-faisceaux se recombinant au niveau de la zone d'interaction avec le matériau à traiter. En conséquence, ces sous-faisceaux étant cohérents, il existe obligatoirement des phénomènes de diffraction et d'interférence. Dans les études citées en référence, nous avons toujours négligé la structure interférentielle de la tache obtenue, c'est-à-dire que nous avons toujours estimé que l'influence de cet effet était négligeable du point de vue des traitements thermiques. Il est intéressant de confirmer ce point. En ce qui concerne les franges de diffraction, leur amplitude et leur périodicité sont si importantes que l'on peut légitimement s'interroger sur les champs de température qui en résultent.

Bien entendu, faire une discussion dans un cas général de la répartition de température résultant de l'interaction laser-matière est très compliqué. L'objet du présent travail consistera donc essentiellement à faire une étude comparative des répartitions de température obtenues en utilisant soit un faisceau primaire gaussien, soit un faisceau transformé par un de nos dispositifs avec un réglage donné, dans le cas de matériaux de différentes natures considérés semi-infinis et homogènes. En outre, pour simplifier, nous admettrons que les caractéristiques thermiques des matériaux retenus sont indépendantes de la température. Bien que simplificatrices, ces hypothèses doivent permettre d'accéder à une description réaliste des champs de température pouvant être obtenus avec différentes répartitions d'énergie sur des matériaux de natures diverses et selon les conditions de l'interaction rayonnement-matériaux. Cela doit permettre de qualifier nos dispositifs vis-à-vis de tel ou tel type d'application.

\section{Echauffement d'un milieu semi-infini par un flux de rayonnement.}

Considérons le milieu semi-infini et la surface à éclairer constituée par le plan $x \mathrm{O} y$ et soit $z$ l'axe perpendiculaire à cette surface et orienté dans le sens du rayonnement. La densité de puissance incidente est supposée très grande devant les pertes par rayonnement pour pouvoir négliger ces dernières. Alors, la condition aux limites à la frontière $z=0$ s'écrit :

$$
\left(\frac{\partial T}{\partial z}\right)_{z=0}=0
$$

Le rayonnement incident est supposé absorbé en surface avec le facteur d'absorption $a$ $(0<a<1)$.

La résolution de l'équation de la chaleur dans ces conditions peut être alors considérée comme la limite, quand la distance de pénétration optique tend vers zéro, de la solution d'un problème d'absorption en volume dans un milieu infini avec sources volumiques de chaleur $p(x, y, z, t)$ symétriques par rapport à $z=0[5]$. 
Pour un faisceau fixe, c'est-à-dire avec une puissance totale et une répartition spatiale d'énergie indépendantes du temps $t$, on peut exprimer la puissance volumique absorbée par le matériau :

$$
p(x, y, z, t)=a I_{0}(x, y, t) \delta(z)=a I_{0}(t) H(x, y) \delta(z) .
$$

Avec, $H(x, y)$, la fonction de répartition énergétique $(0<H(x, y)<1)$ et $\delta(z)$, une fonction Delta. D'après la définition de la puissance volumique et compte tenu de la direction de propagation du faisceau, le facteur $I_{0}$ (éclairement de la face avant au point $O$ ) est, pour une puissance indicente $P_{0}$ donnée, déterminé par l'expression :

$$
\begin{aligned}
2 P_{0}(t)=I_{0}(t) \int_{-\infty}^{+\infty} \mathrm{d} x^{\prime} \int_{-\infty}^{+\infty} \mathrm{d} y^{\prime} \int_{-\infty}^{+\infty} \mathrm{d} z^{\prime} \delta\left(z^{\prime}\right) H\left(x^{\prime}, y^{\prime}\right)= \\
=I_{0}(t) \int_{-\infty}^{+\infty} \mathrm{d} x^{\prime} \int_{-\infty}^{+\infty} \mathrm{d} y^{\prime} H\left(x^{\prime}, y^{\prime}\right) .
\end{aligned}
$$

La variation de température observée en un point $(x, y, z)$ du milieu semi-infini à l'instant $\tau$ s'exprime [5]:

$$
\begin{aligned}
\Delta T(x, y, z, \tau)=\int_{0}^{\tau} \mathrm{d} t \int_{-\infty}^{+\infty} \mathrm{d} x^{\prime} \int_{-\infty}^{+\infty} \mathrm{d} z^{\prime} & \frac{p\left(x^{\prime}, y^{\prime}, z^{\prime}, t\right)}{8 k \alpha^{1 / 2}[\pi(\tau-t)]^{3 / 2}} \times \\
& \times \exp \left[-\frac{\left(x-x^{\prime}\right)^{2}+\left(y-y^{\prime}\right)^{2}+\left(z-z^{\prime}\right)^{2}}{4 \alpha(\tau-t)}\right]
\end{aligned}
$$

avec: $k$ et $\alpha$ conductivité et diffusivité thermiques du matériau.

En substituant (2.1) dans (2.3) et en utilisant la propriété de la fonction Delta, la température en un point quelconque du milieu semi-infini, dans l'hypothèse d'absorption de surface s'écrit :

$$
\begin{aligned}
\Delta T(x, y, z, \tau)=\int_{0}^{\tau} \mathrm{d} t \frac{a I_{0} \exp \left(-\frac{z^{2}}{4 \alpha(\tau-t)}\right)}{8 k \alpha^{1 / 2}[\pi(\tau-t)]^{3 / 2}} \int_{-\infty}^{+\infty} \mathrm{d} x^{\prime} \int_{-\infty}^{\infty} \mathrm{d} y^{\prime} H\left(x^{\prime}, y^{\prime}\right) \times \\
\times \exp \left[-\frac{\left(x-x^{\prime}\right)^{2}+\left(y-y^{\prime}\right)^{2}}{4 \alpha(\tau-t)}\right] .
\end{aligned}
$$

L'élévation de température en face avant s'écrit :

$$
\begin{aligned}
& \Delta T(x, y, 0, \tau)=\int_{0}^{\tau} \mathrm{d} t \frac{a I_{0}}{8 k \alpha^{1 / 2}[\pi(\tau-t)]^{3 / 2}} \int_{-\infty}^{\infty} \mathrm{d} x^{\prime} \int_{-\infty}^{+\infty} \mathrm{d} y^{\prime} H\left(x^{\prime}, y^{\prime}\right) \times \\
& \times \exp \left[-\frac{\left(x-x^{\prime}\right)^{2}+\left(y-y^{\prime}\right)^{2}}{4 \alpha(\tau-t)}\right]
\end{aligned}
$$

L'expression précédente peut être facilement généralisée au cas du faisceau mobile.

Après un choix convenable du repérage des coordonnées, nous pouvons toujours considérer que le mouvement relatif du faisceau par rapport au matériau s'effectue à une vitesse $v$ dans la direction $x$ et donc écrire la fonction de répartition d'énergie du faisceau sous la forme $H(x-v t, y)$. Dans ces conditions si nous admettons que l'effet du déplacement du faisceau par rapport au matériau est équivalent à celui d'un faisceau fixe ayant une répartition d'énergie variable au cours du temps, tous les résultats obtenus précédemment peuvent être immédiatement généralisés. Par suite nous obtenons : 


$$
\begin{aligned}
& \Delta T(x, y, z, \tau)=\int_{0}^{\tau} \mathrm{d} t \frac{a I_{0} \exp \left(-\frac{z^{2}}{4 \alpha(\tau-t)}\right)}{8 k \alpha^{1 / 2}[\pi(\tau-t)]^{3 / 2}} \int_{-\infty}^{\infty} \mathrm{d} x^{\prime} \int_{-\infty}^{+\infty} \mathrm{d} y^{\prime} H\left(x^{\prime}-v t, y^{\prime}\right) \times \\
& \times \exp \left[-\frac{\left(x-x^{\prime}\right)^{2}+\left(y-y^{\prime}\right)^{2}}{4 \alpha(\tau-t)}\right] .
\end{aligned}
$$

Le résultat pour $v=0$ de l'expression (2.5) correspondant à celui du faisceau fixe, nous n'utiliserons donc que cette dernière expression pour la suite des calculs.

\section{Analyse des effets thermiques induits par un dispositif d'homogénéisation.}

3.1 SYSTÈmE OPTIQUE D'HOMOgÉnÉISATION. - Comme le principe d'homogénéisation du faisceau reste pratiquement le même dans tous nos dispositifs, pour simplifier la présente analyse nous n'étudierons ici que la forme la plus simple (correspondant au dispositif $L_{2}$ [4] schématisé Fig. 1). Ce dispositif est composé d'un miroir prismatique à deux faces et de deux

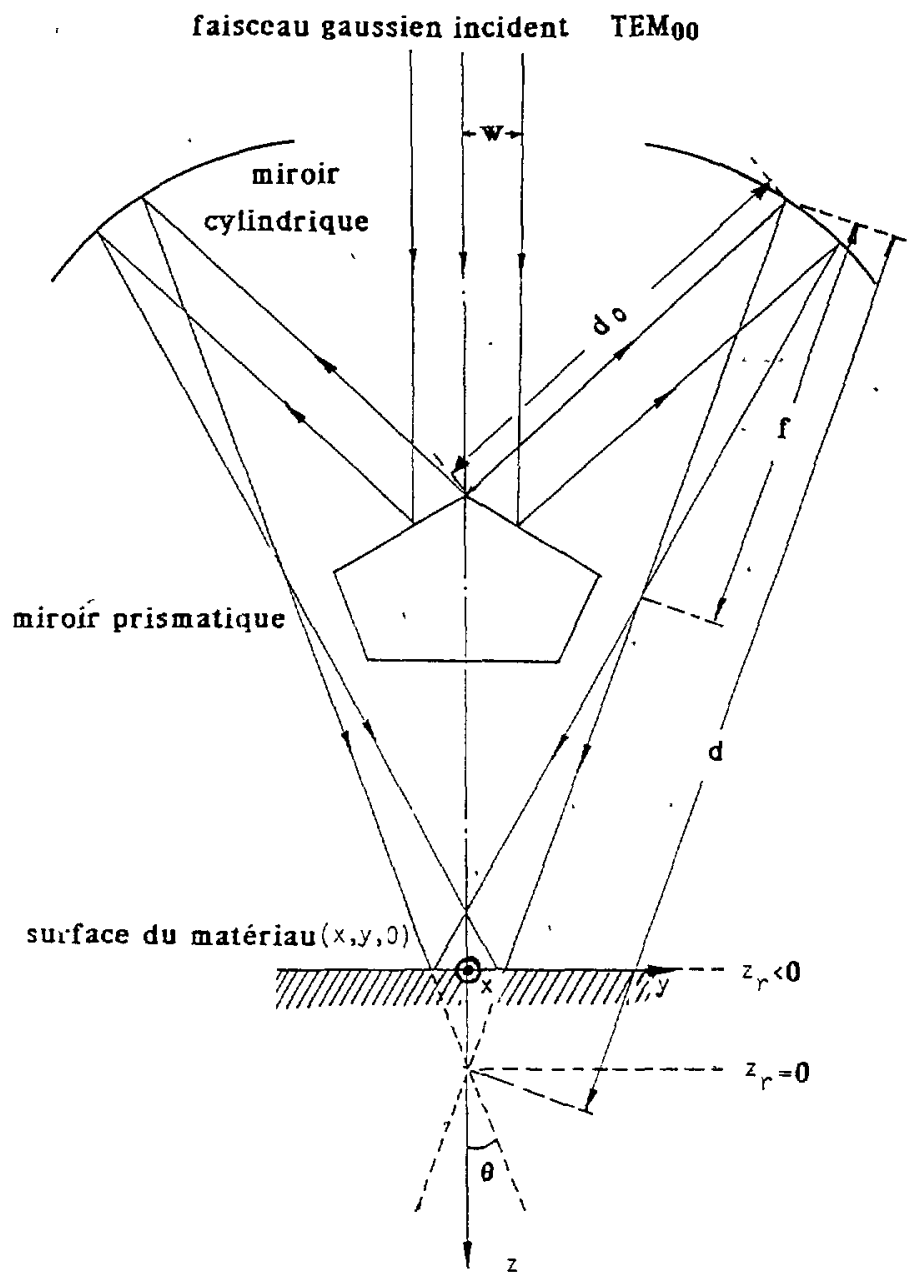

Fig. 1. - Schéma de principe du dispositif d'homogénéisation en «bande gaussienne ".

[Schematic diagram of the studied optic device.] 
miroirs cylindriques. Il permet d'obtenir une «bande gaussienne » par simple recombinaison de deux sous-faisceaux à partir d'un faisceau primaire gaussien TEM $_{00}$ [2].

Le fait de subdiviser le faisceau primaire introduit des phénomènes de diffraction avec des caractéristiques fonction de la position du plan d'observation mais dont l'amplitude est toujours d'autant plus marquée que l'on se situe plus près de la « ligne de coupe » du faisceau subdivisé. En outre, le faisceau primaire étant cohérent, la superposition éventuelle de sousfaisceaux après subdivision est susceptible de donner naissance à des phénomènes d'interférence. Il en résulte donc un double réseau de franges.

Afin d'avoir une image plus claire de la transformation du faisceau gaussien par le dispositif, nous donnons, figures $2 a, b$, les deux répartitions d'énergie correspondant respectivement au faisceau avant et après transformation en "bande gaussienne » (nota : on a seulement tenu compte sur cette figure du phénomène de diffraction; les interfranges correspondant au phénomène d'interférence, étant trop réduites pour être représentées).

Pour calculer la répartition de température induite par ce dispositif $L_{2}$, il nous faut exprimer correctement la puissance volumique au niveau de la surface traitée. Si l'on suppose

a)

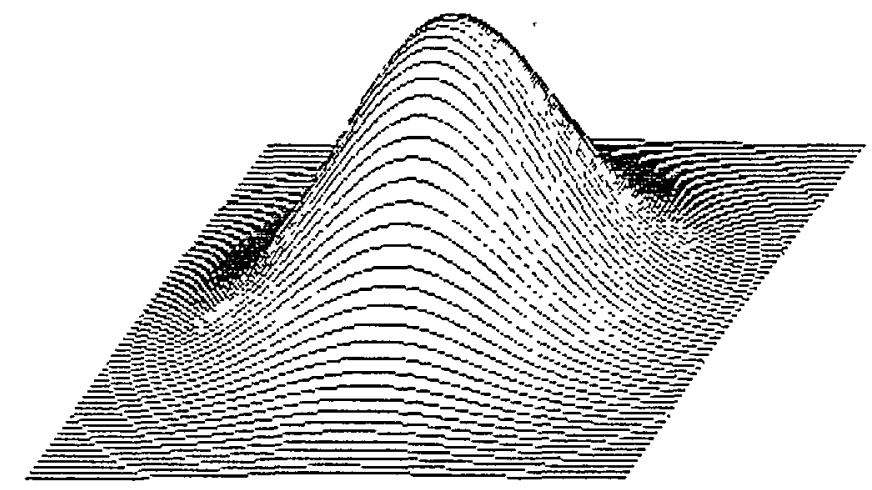

b)

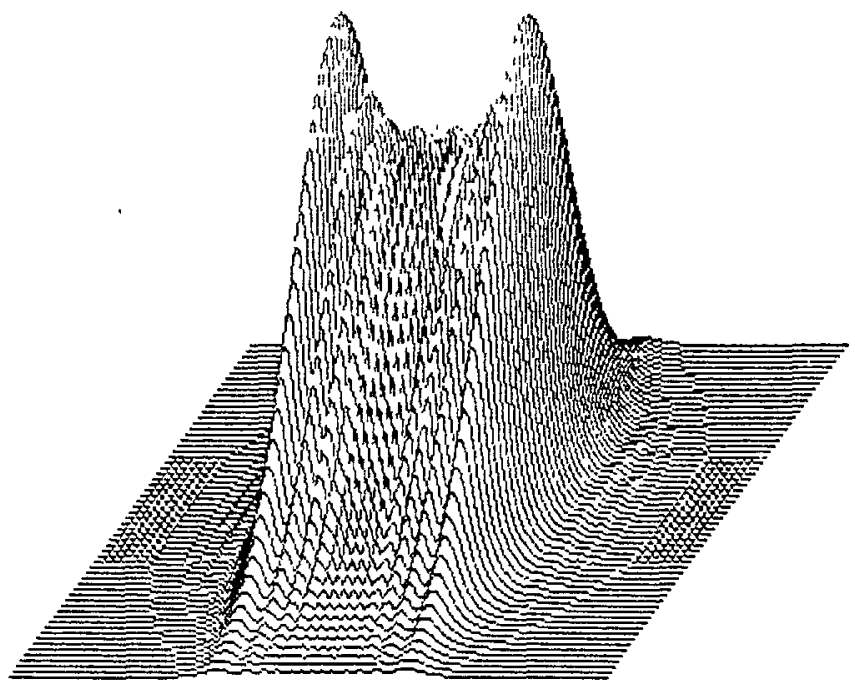

Fig. 2. - Comparaison de la forme des répartitions énergétiques correspondant à un faisceau gaussien avant (2a) et après (2b) sa transformation par le dispositif de la figure 1 .

[Comparison of energy distribution shape before (2a) and after (2b) transformation by the optic device.] 
que le faisceau gaussien incident a un rayon $w$ (à $1 / e^{2}$ ) et une puissance totale $P_{0}$, en utilisant le système de coordonnées avec les définitions qui sont indiquées sur la figure 1, et en repartant de l'équation (2.1), on peut montrer d'après [4] que :

$$
I_{0}(x, y, t)=\frac{4 P_{0}}{\pi w^{2}} E(y) \exp \left[-2 \frac{(x-v t)^{2}}{w^{2}}\right] .
$$

$E(y)$ est la fonction de répartition d'énergie totale pouvant se décomposer en deux termes $E_{\mathrm{g}}(y)$ et $E_{\imath}(y) . E_{\mathrm{g}}(y) \exp \left[-2 \frac{(x-v t)^{2}}{w^{2}}\right]$ est représentatif de la superposition des éclairements moyens des deux sous-faisceaux qu'on obtiendrait dans l'hypothèse de l'optique géométrique, tandis que $E_{i}(y) \exp \left[-2(x-v t)^{2} / w^{2}\right]$ est représentatif de la structure des interférences [6]

$$
\begin{aligned}
& E_{\mathrm{g}}(y)=\frac{|U(y)|^{2}+|U(-y)|^{2}}{M_{y}^{2} \lambda\left(d+z_{\mathrm{r}} \cos \theta-y \sin \theta+d_{0} M_{y}\right)} \\
& E_{1}(y)=\frac{U^{*}(y) U(-y)+U^{*}(-y) U(y)}{M_{y}^{2} \lambda\left(d+z_{\mathrm{r}} \cos \theta-y \sin \theta+d_{0} M_{y}\right)}
\end{aligned}
$$

avec :

$d_{0}$ distance objet virtuel (distance entre l'arête du prisme et le plan de la lentille équivalente de focale $f$ ).

$z_{\mathrm{r}}$ distance séparant le plan d'observation de l'intersection de l'axe optique de la lentille équivalente avec le faisceau primaire.

$M_{y}$ grandissement transversal selon la direction $y$

$$
M_{y}=1-\frac{d+z_{\mathrm{r}} \cos \theta-y \sin \theta}{f}
$$

et $U(y)$ amplitude de la vibration lumineuse, c'est-à-dire du champ électrique complexe dans le plan d'observation

$$
\begin{aligned}
U(y)=\int_{-z_{\mathrm{r}} \sin \theta}^{2 M_{y} w-z_{\mathrm{r}} \sin \theta} \exp \left[-\frac{\left(\beta+z_{\mathrm{r}} \sin \theta\right)^{2}}{M_{y}^{2} w^{2}}\right] \times \\
\quad \times \exp \left[j \frac{\pi(\beta-y \cos \theta)^{2}}{\lambda M_{y}\left(d+z_{\mathrm{r}} \cos \theta-y \sin \theta+d_{0} M_{y}\right)}\right] \mathrm{d} \beta
\end{aligned}
$$

(une fois le changement de variable $\beta=y-z_{\mathrm{r}} \sin \theta$ effectué et avec $\lambda$, longueur d'onde du faisceau laser.)

3.2 EXPRESSION DU CHAMP DE TEMPERATURE INDUIT PAR UNE RÉPARTITION EN «BANDE GAUSSIENNE ». - A partir des discussions précédentes et d'après les expressions (3.1) et (2.5), on obtient alors :

$$
\begin{array}{r}
\Delta T=\int_{0}^{\tau} \mathrm{d} t \frac{a P_{0} \exp \left(-\frac{z^{2}}{4 \alpha(\tau-t)}\right)}{2 k \alpha^{1 / 2} \pi w^{2}[\pi(\tau-t)]^{3 / 2}} \int_{-\infty}^{+\infty} \mathrm{d} x^{\prime} \int_{-\infty}^{+\infty} \mathrm{d} y^{\prime}\left[E_{\mathrm{g}}\left(y^{\prime}\right)+E_{\mathrm{i}}\left(y^{\prime}\right)\right] \times \\
\times \exp \left[-2 \frac{\left(x^{\prime}-v t\right)^{2}}{w^{2}}\right] \exp \left[-\frac{\left(x-x^{\prime}\right)^{2}+\left(y-y^{\prime}\right)^{2}}{4 \alpha(\tau-t)}\right] .
\end{array}
$$

Il est clair que le calcul numérique de l'expression précédente est compliqué. Pour diminuer la difficulté nous allons effectuer quelques simplifications avant le calcul. 
3.2.1. Influence du phénomène d'interférence sur le champ de température. - Pour simplifier l'estimation de l'influence des interférences nous considérons que la répartition d'énergie de la tache est déterminée approximativement par la superposition cohérente de deux ondes cylindriques centrées respectivement aux lignes-focales des deux miroirs cylindriques [6]. Dans ces conditions (voir Annexe A) $E_{\mathrm{g}}(y)$ et $E_{1}(y)$ peuvent être identifiés à $H_{\mathrm{g}}(y)$ et $H_{1}(y)$ définis par:

$$
\begin{gathered}
H_{\mathrm{g}}(y)=\left\{\begin{array}{l}
\frac{1}{M_{y}} \exp \left[-\frac{2\left(y \cos \theta+z_{\mathrm{r}} \sin \theta\right)^{2}}{M_{y}^{2} w^{2}}\right] \text { pour }\left(y \leqslant z_{\mathrm{r}} \operatorname{tg} \theta\right) \\
\frac{1}{M_{y}} \exp \left[-\frac{2\left(y \cos \theta-z_{\mathrm{r}} \sin \theta\right)^{2}}{M_{y}^{2} w^{2}}\right] \text { pour }\left(y \geqslant-z_{\mathrm{r}} \operatorname{tg} \theta\right) \\
\frac{1}{M_{y}}\left\{\exp \left[-\frac{2\left(y \cos \theta-z_{\mathrm{r}} \sin \theta\right)^{2}}{M_{y}^{2} w^{2}}\right]+\exp \left[-\frac{2\left(y \cos \theta+z_{\mathrm{r}} \sin \theta\right)^{2}}{M_{y}^{2} w^{2}}\right]\right\} \\
H_{1}(y)=\operatorname{rect}\left(\frac{y}{-2 z_{\mathrm{r}} \operatorname{tg} \theta}\right) \frac{2}{M_{y}} \exp \left[-2 \frac{y^{2} \cos ^{2} \theta+z_{\mathrm{r}}^{2} \sin ^{2} \theta}{M_{y}^{2} w^{2}}\right] \cos \left(\frac{2 \pi}{F_{\mathrm{i}}} y\right)
\end{array}\right.
\end{gathered}
$$

avec $F_{1}=\frac{\lambda}{2 \sin \theta}$ et $M_{y}$ le grandissement transversal selon $y$.

D'après les propriétés de linéarité des intégrales, nous pouvons calculer isolément les variations induites par les deux termes. Le calcul isolé des variations de température induites par ces deux termes permet de comparer les effets thermiques résultants et donc d'évaluer le rôle joué par l'effet d'interférence. Afin de faciliter cette comparaison, les simplifications suivantes sont effectuées:

a) La vitesse de déplacement relatif $v$ entre le laser et le matériau à traiter, ainsi que le grandissement transversal $M_{y}$, n'influençant pas la valeur relative de ces deux effets, on se place dans la situation où $v=0$ et $M_{y}=1$;

b) L'expression de $H_{\mathrm{i}}(y)$ montre que la structure interférentielle est constituée de franges ayant un intervalle constant de l'ordre de quelques longueurs d'onde, avec un maximum d'amplitude se situant au centre de la tache recombinée. Pour évaluer le maximum d'influence possible, nous ne considérons que la situation pour laquelle l'amplitude de la structure interférentielle est maximale, ce qui se produit pour $\exp \left[-2(x-v t)^{2} / w^{2}\right]=1$ et $\exp \left[-2\left(y^{2} \cos ^{2} \theta+z_{\mathrm{r}}^{2} \sin ^{2} \theta\right) /\left(M_{y}^{2} w^{2}\right)\right]=1$. En outre, nous étendons cette structure interférentielle à tout le plan d'observation, c'est-à-dire que nous supprimons le terme rect $\left[y /\left(-2 z_{\mathrm{r}} \operatorname{tg} \theta\right)\right]$, ce qui facilite notablement le calcul, mais l'intervalle entre franges d'interférences étant très petit, la contribution de cette partie rajoutée pour effectuer l'intégration est négligeable du fait des alternances de signe. Par conséquent, les expressions (3.5a) et $(3.5 b)$ se réduisent à :

$$
H_{\mathrm{g}}(y)=\left\{\begin{array}{l}
\exp \left[-\frac{2\left(y \cos \theta+z_{\mathrm{r}} \sin \theta\right)^{2}}{w^{2}}\right] \quad \text { pour }\left(y \leq z_{\mathrm{r}} \operatorname{tg} \theta\right) \\
\exp \left[-\frac{2\left(y \cos \theta-z_{\mathrm{r}} \sin \theta\right)^{2}}{w^{2}}\right] \quad \text { pour }\left(y \geqslant-z_{\mathrm{r}} \operatorname{tg} \theta\right) \\
\exp \left[-\frac{2\left(y \cos \theta-z_{\mathrm{r}} \sin \theta\right)^{2}}{w^{2}}\right]+\exp \left[-\frac{2\left(y \cos \theta+z_{\mathrm{r}} \sin \theta\right)^{2}}{w^{2}}\right] \\
\text { pour }\left(z_{\mathrm{r}} \operatorname{tg} \theta<y<-z_{\mathrm{r}} \operatorname{tg} \theta\right)
\end{array}\right.
$$




$$
H_{1}(y)=2 \cos \left(\frac{2 \pi}{F_{1}} y\right)
$$

c) L'évaluation du champ de température à l'intérieur du matériał pouvant se déduire de celui observé en surface, nous nous intéresserons surtout aux variations de température superficielle, c'est-à-dire lorsque $\exp \left[-z^{2} /(4 \alpha(\tau-t))\right]=1$ (soit $z$ tend vers zéro).

Avec ces trois simplifications et en utilisant l'expression (2.5), on a trouvé les expressions permettant de calculer isolément l'effet thermique observé en surface et résultant de la superposition des éclairements $\Delta T_{\mathrm{g}}$;

$$
\Delta T_{\mathrm{g}}=\int_{0}^{\tau} \mathrm{d} t \frac{a P_{0} \exp \left(-\frac{2 x^{2}}{8 \alpha(\tau-t)+w^{2}}\right)}{k \pi^{2} w(\tau-t) \sqrt{8 \alpha(\tau-t)+w^{2}}} \int_{-\infty}^{+\infty} \mathrm{d} y^{\prime} H_{\mathrm{g}}\left(y^{\prime}\right) \exp \left[-\frac{\left(y-y^{\prime}\right)^{2}}{4 \alpha(\tau-t)}\right]
$$

qui, pour $\tau$ suffisamment petit (c'est-à-dire $\tau \ll w^{2} / \alpha$ ), s'exprime par

$$
\Delta T_{\mathrm{g}}=\frac{4 a P_{0} \exp \left(-\frac{2 x^{2}}{w^{2}}\right) H_{\mathrm{g}}(y)}{\pi^{3 / 2} w^{2} k} \sqrt{\alpha \tau}
$$

ainsi que celui résultant de la structure interférentielle $\Delta T_{\mathrm{i}}$ :

$$
\Delta T_{1}=\frac{2 F_{\mathrm{i}} a P_{\cdot_{0}} \cos \left(\frac{2 \pi}{F_{1}} y\right)}{k \pi^{2} w^{2}} \operatorname{erf}\left(\frac{2 \pi \sqrt{\alpha \tau}}{F_{\mathrm{i}}}\right) .
$$

Par rapport à l'évolution moyenne (ou "géométrique») $\Delta T_{\mathrm{g}}$, et du fait du terme $\cos \left(2 \pi y / F_{1}\right), \Delta T_{1}$ peut être considéré comme un effet perturbateur positif ou négatif; nous ne considérerons donc que son amplitude maximale

$$
\left|\Delta T_{1}\right|_{\max }=\frac{2 F_{\mathrm{i}} a P_{0}}{k \pi^{2} w^{2}} \operatorname{erf}\left(\frac{2 \pi \sqrt{\alpha \tau}}{F_{1}}\right) .
$$

Cette amplitude maximale croît au cours du temps̀ par l'intermédiaire de la fonction « erf », mais on sait que erf $(u)$ tend très vite vers 1 (dès que $u>2$, soit $\tau>F_{1}^{2} /\left(\alpha \pi^{2}\right)$ ), $\alpha$ est fonction de la nature du matériau, pour les matériaux conducteurs (métaux) $\alpha>10 \mathrm{~mm}^{2} / \mathrm{s}$, pour les matériaux isolants (céramiques) $\alpha<1 \mathrm{~mm}^{2} / \mathrm{s}, F_{1}$ est toujours de l'orḋe de grandeur de quelques $\lambda$ (soit quelques dizaines de $\mu \mathrm{m}$ dans le cas d'une source laser $\mathrm{CO}_{2}$ avec $\lambda=10,6 \mu \mathrm{m}$, et plus précisément $F_{1}=\lambda /\left(2 \sin \left(13,2^{\circ}\right)\right)$ dans le cas de notre dispositif (soit $F_{1} \approx 20 \mu \mathrm{m}$ ). L'amplitude $\Delta T_{1}$ arrive donc très rapidement à son maximum. Par ailleurs $w$ étant généralement de l'ordre de quelques millimètres, cela permet ainsi de choisir l'expression (3.6b) pour comparer $\Delta T_{1}$ et $\Delta T_{\mathrm{g}}$. En outre, on peut considérer au centre d'une tache en "bande gaussienne » que la fonction $H_{\mathrm{g}}(y) \exp \left(-2 x^{2} / w^{2}\right)$ correspond à une répartition énergétique « homogène » et est égale à l'unité. Par conséquent

$$
\frac{\left|\Delta T_{\mathrm{i}}\right|_{\max }}{\Delta T_{\mathrm{g}}} \approx \frac{F_{1}}{2 \sqrt{\pi \alpha \tau}} .
$$

- Dès que

$$
\tau>\tau_{1}=8 F_{\mathrm{i}}^{2} / \alpha
$$


Nous constatons alors que $\left|\Delta T_{1}\right|_{\text {max }} / \Delta T_{\mathrm{g}}<0,1$ et donc que l'influence de l'effet d'interférence peut alors être négligée.

Il reste encore à préciser l'ordre de grandeur de $\tau_{1}$, qui dépend de la nature des matériaux :

$$
\begin{aligned}
& \tau_{1} \text { (conducteur) }<3 \times 10^{-4} \mathrm{~s} \\
& \tau_{\mathrm{i}^{\prime}}(\text { isolant })
\end{aligned}
$$

Etant donné les temps d'interaction habituellement rencontrés dans les traitements superficiels des matériaux (de l'ordre de la fraction de seconde à quelques secondes [5]) ces valeurs de $\tau_{1}$ montrent que les perturbations liées au phénomène d'interférence peuvent généralement être négligées (tout particulièrement dans le cas du traitement des matériaux métalliques).

3.2.2 Influence du phénomène de diffraction sur le champ de température. - Pour nos dispositifs, l'intervalle des franges de diffraction est généralement plus important que celui des franges d'interférence. La variation de la répartition d'énergie résultant de ce phénomène est nette, surtout au bord de la tache (voir Fig. 2b). Donc, il est nécessaire d'évaluer son influence thermique. La description mathématique rigoureuse du réseau de franges de diffraction (dont un exemple est donné dans [1]) est assez complexe mais la pseudopériodicité du phénomène doit permettre, comme dans le cas de la discussion précédente, d'évaluer l'intensité des effets résultants à partir d'une expression de la forme $H_{\mathrm{d}}(y)=$ $A \cos \left(2 \pi y / F_{\mathrm{d}}\right)$ que l'on considérera comme une perturbation se superposant à la répartition d'énergie prévue par l'optique géométrique. Comme les franges les plus fortes se situent au bord de la tache, pour simplifier, nous considérerons la période spatiale $F_{\mathrm{d}}$ comme la moyenne des intervalles des dix premières franges de diffraction. D'après la référence [4] on obtient alors :

$$
\begin{aligned}
F_{\mathrm{d}}=\frac{1}{20 \cos \theta} \sum_{n=1}^{10} \sqrt{\lambda M_{y}\left(d+z_{\mathrm{r}} \cos \theta-y \sin \theta+d_{0} M_{y}\right)} \times \\
\times\left[\sqrt{\frac{(4 n+1)}{2}}+\sqrt{2 n+1}-\sqrt{\frac{(4 n-3)}{2}}-\sqrt{2 n-1}\right] .
\end{aligned}
$$

Soit $F_{\mathrm{d}} \simeq 0,6 \mathrm{~mm}$ dans le cas de notre dispositif utilisé avec une source laser $\mathrm{CO}_{2}$ pour $M_{y}=1$.

Pair ailleurs, d'après la forme calculée de la répartition d'énergie de diffraction (voir Fig. 2b ou 5a2) et également d'après des études expérimentales réalisées [4] nous pouvons estimer que l'amplitude maximale de cette oscillation simulée est de l'ordre de la moitié de l'amplitude de la répartition "géométrique" $(A=0,5)$. Ainsi on obtient de manière analogue :

$$
\left|\Delta T_{\mathrm{d}}\right|_{\max }=\frac{F_{\mathrm{d}} \rho P_{0}}{2 k \pi^{2} w^{2}} \operatorname{erf}\left(\frac{2 \pi \sqrt{\alpha \tau}}{F_{\mathrm{d}}}\right) .
$$

- Dès que

$$
\tau>\tau_{\mathrm{d}}=25 F_{\mathrm{d}}^{2} / 16 \pi \alpha .
$$

Le rapport $\left|\Delta T_{\mathrm{d}}\right|_{\max } / \Delta T_{\mathrm{g}}$ est inférieur à $10 \%$ et les perturbations introduites par la diffraction peuvent alors être négligées. En considérant toujours nos deux classes de matériaux (pour $F_{\mathrm{d}}=0,6 \mathrm{~mm}$ ) on aboutit donc à : 


$$
\begin{aligned}
& \tau_{\mathrm{d}}(\text { conducteur })<2 \times 10^{-2} \mathrm{~s} \\
& \tau_{\mathrm{d}^{\prime}}(\text { isolant })
\end{aligned}
$$

Par conséquent, les effets des perturbations introduites par la diffraction ne peuvent plus être systématiquement négligés. Pour un certain nombre de traitements sur les matériaux métalliques ce sera encore possible mais ce ne le sera plus pour des matériaux de type céramique.

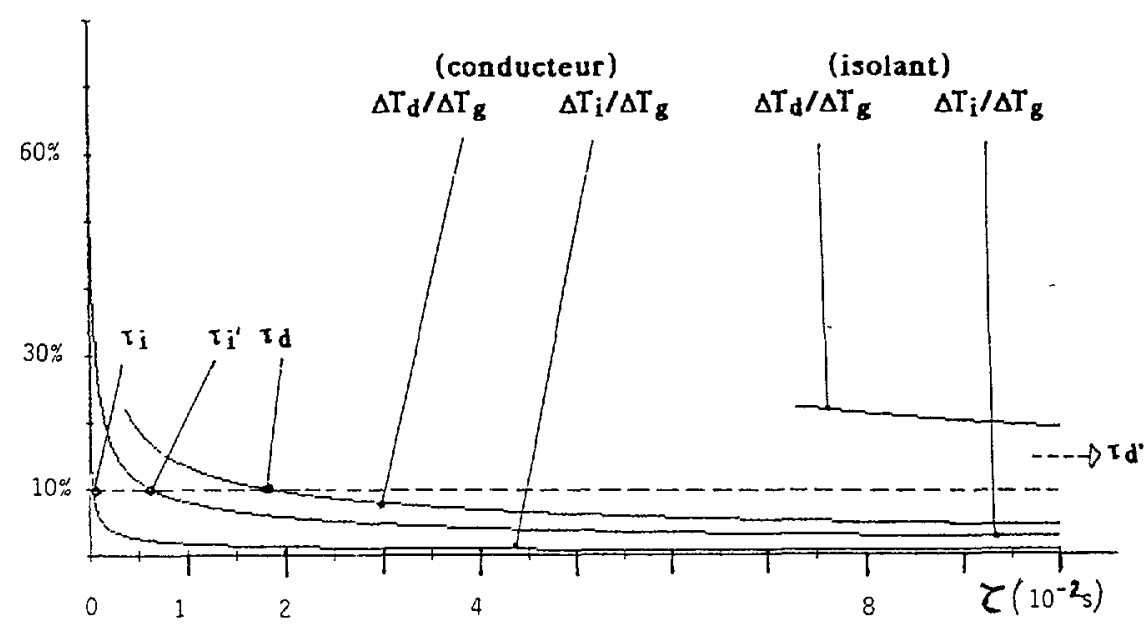

Fig. 3. - Comparaison des effets thermiques induits par les phénomènes d'interférence et de diffraction pour deux types de matériaux isolant ou conducteur.

[Comparison of thermal effects induced by interference and diffraction phenomena for two kinds of materials (conducting and insulating).]

En résumé, afin de comparer les deux effets thermiques perturbateurs introduits par les phénomènes d'interférence et de diffraction nous présentons, figure 3 , les courbes donnant les variations en fonction du temps des rapports $\Delta T_{\mathrm{i}} / \Delta T_{\mathrm{g}}$ et $\Delta T_{\mathrm{d}} / \Delta T_{\mathrm{g}}$ pour les deux classes de matériaux conducteurs et isolants. Et figure $4 \mathrm{a}, \mathrm{b}$ nous donnons, comme justification expérimentale, les impacts des deux sous-faisceaux obtenus sur un papier thermique après une irradiation de $20 \mathrm{~ms}$ [4] avant et après leur recombinaison. Il est hors de doute que la diffusivité thermique du papier est très faible $\left(\alpha<0,1 \mathrm{~mm}^{2} \mathrm{~s}^{-1}\right.$, ce qui conduit à $\tau_{1^{\prime}}>3 \times 10^{-2} \mathrm{~s}$ et $\tau_{\mathrm{d}^{\prime}}>2 \mathrm{~s}$ ) et pourtant on ne détecte aucun effet d'interférence dans cet essai tandis que l'on observe clairement des franges de diffraction. Par conséquent, dans toutes études concernant les traitements laser, que ce soit sur les métaux, ou sur des matériaux mauvais conducteurs, on pourra négliger l'influence du phénomène d'interférence sur la répartition de température (et les autres effets en découlant).

\section{Calcul numérique complet dans le cas du dispositif en « bande gaussienne ».}

4.1 EVALUATION DU CHAMP DE TEMPERATURE SUPERFICIELLE INDUIT PAR UN SOUS-FAISCEAU. - D'après les discussions précédentes, l'influence du phénomène d'interférence est donc négligée, et l'expression (3.4) se réduit finalement à 

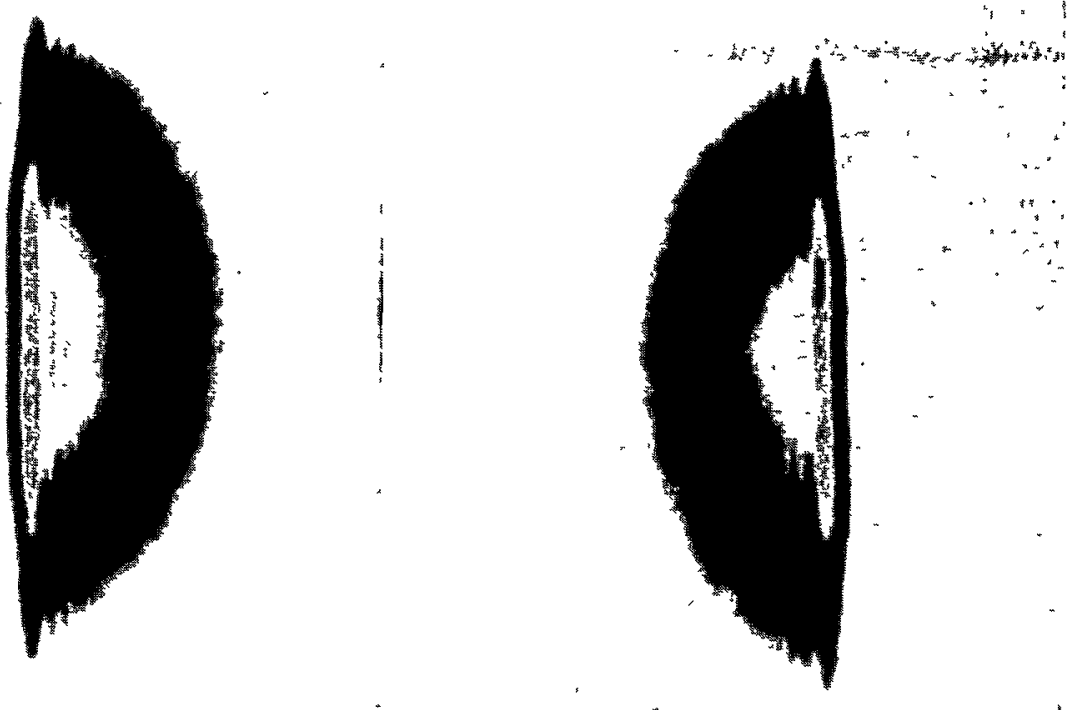

a)

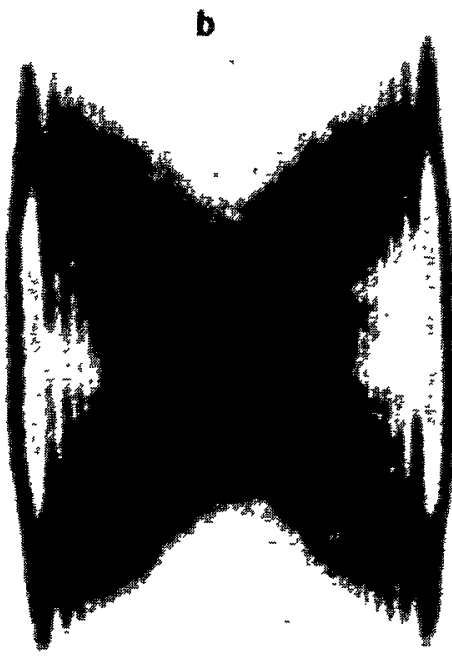

b)

Fig. 4. - Mise en évidence expérimentale de la prépondérance de l'effet de diffraction sur l'effet d'interférence au moyen d'un papier thermique irradié pendant $20 \mathrm{~ms}$. Les deux demi-faisceaux avant (4a) et après recombinaison (4b).

[Experimental views showing the thermal effects induced by diffraction and interference phenomena on an insulating material; the two half-beams before (4a) and after recombination (4b).] 


$$
\begin{aligned}
\Delta T(x, y, 0, \tau)=\int_{0}^{\tau} \mathrm{d} t & \frac{a P_{0} \exp \left(-\frac{2(x-v t)^{2}}{8 \alpha(\tau-t)+w^{2}}-\frac{z^{2}}{4 \alpha(\tau-t)}\right)}{k \pi^{2} w(\tau-t) \sqrt{8 \alpha(\tau-t)+w^{2}}} \times \\
& \times \int_{-\infty}^{+\infty} \mathrm{d} y^{\prime} \frac{\left[\left|U\left(y^{\prime}\right)\right|^{2}+\left|U\left(-y^{\prime}\right)\right|^{2}\right] \exp \left[-\frac{\left(y-y^{\prime}\right)^{2}}{4 \alpha(\tau-t)}\right]}{M_{y}^{2} \lambda\left(d+z_{\mathrm{r}} \cos \theta-y \sin \theta+d_{0} M_{y}\right)}
\end{aligned}
$$

Selon le principe du dispositif $\mathrm{L}_{2^{\prime}}$, on peut faire varier continuement la forme de la répartition d'énergie dans la zone d'interaction en changeant le niveau du plan d'observation (c'est-à-dire en se plaçant à différentes cotes $z_{\mathrm{r}}$ ) et par conséquent induire différentes formes du champ de.température en surface du matériau irradié. Mais il faut noter que les formes du champ de température obtenues avec un seul sous-faisceau dans les différents niveaux d'observation sont similaires à un facteur de grandissement près. De plus, grâce aux propriétés de linéarité de l'intégrale et étant donné la symétrie géométrique des deux sousfaisceaux, on peut, à partir du calcul des effets induits par un seul des sous-faisceaux dans un des niveaux d'observation, simuler les différentes formes possibles de champs de température par de simples combinaisons symétriques.

Par ailleurs, afin de mettre en évidence les différences des champs de température superficielle induits selon les caractéristiques thermiques des matériaux, nous avons retenu un acier et une céramique pour réaliser la comparaison. Les caractéristiques prises pour effectuer les calculs sont les suivantes:

acier : $\alpha=10 \mathrm{~mm}^{2} / \mathrm{s}, k=0,04 \mathrm{~W} / \mathrm{mm} \mathrm{K}$ céramique : $\alpha=0,5 \mathrm{~mm}^{2} / \mathrm{s}^{-1}, k=10^{-3} \mathrm{~W} / \mathrm{mm} \mathrm{K}$

faisceau : $w=8 \mathrm{~mm}, P_{0}=300 \mathrm{~W}, \lambda=10,6 \mu \mathrm{m}$

système optique : $\theta=13,7^{\circ}, d_{0}=50 \mathrm{~mm}, d=206 \mathrm{~mm}, z_{\mathrm{r}}=-5 \mathrm{~mm}$

d'où l'on tire pour l'acier $\tau_{\mathrm{d}} \simeq 0,04 \mathrm{~s}$ et pour la céramique $\tau_{\mathrm{d}^{\prime}} \simeq 0,4 \mathrm{~s}$.

Pour simplifier les comparaisons, on a considéré un facteur d'absorption $a$ égal à 0,5 dans les deux cas.

Afin de mettre en évidence la structure de la répartition d'énergie dans un sous-faisceau, sur la figure 5a sont schématisées respectivement deux coupes axiales correspondant aux répartitions théoriques déduites de l'optique ondulatoire (Fig. 5a2) et de l'optique géométrique (Fig. 5a1). Les résultats obtenus après un temps d'irradiation $\tau=0,5 \mathrm{~s}$ en partant de ces deux types de répartitions théoriques sont présentés figures $5 \mathrm{~b}$ et $5 \mathrm{c}$. Evidemment, les différences d'effusivité $(k / \sqrt{\alpha})$ des deux matériaux conduisent à des champs de température d'amplitudes très différentes.

Ces calculs confirment qu'on ne peut presque pas observer l'influence de la diffraction sur la répartition de température pour l'acier. Et il est intéressant de constater que les deux répartitions d'énergie établies à partir soit de l'optique ondulatoire soit de l'optique géométrique conduisent sensiblement au même résultat. C'est-à-dire que l'on peut assimiler la répartition d'énergie de.la tache à celle prévue par l'optique géométrique lorsque le temps d'interaction est assez grand et que le matériau possède une bonne diffusivité thermique (c'est-à-dire pour $\tau \gg 25 F_{\mathrm{d}}^{2} / 16 \pi \alpha$ ). Cela doit permettre de simplifier notablement ${ }^{2}$-le traitement des informations expérimentales.

Par contre, dans le cas du matériau céramique isolant pour lequel $\tau=0,5 \mathrm{~s}$ est de l'ordre de $\tau_{\mathrm{d}^{\prime}}$, il existe encore une influence de la diffraction sur la répartition de température (voir Fig. 5c2). On constate bien évidemment que l'influence la plus importante se situe en bordure de tache. Donc, l'effet de la diffraction sera fonction des conditions expérimentales. Cet effet 

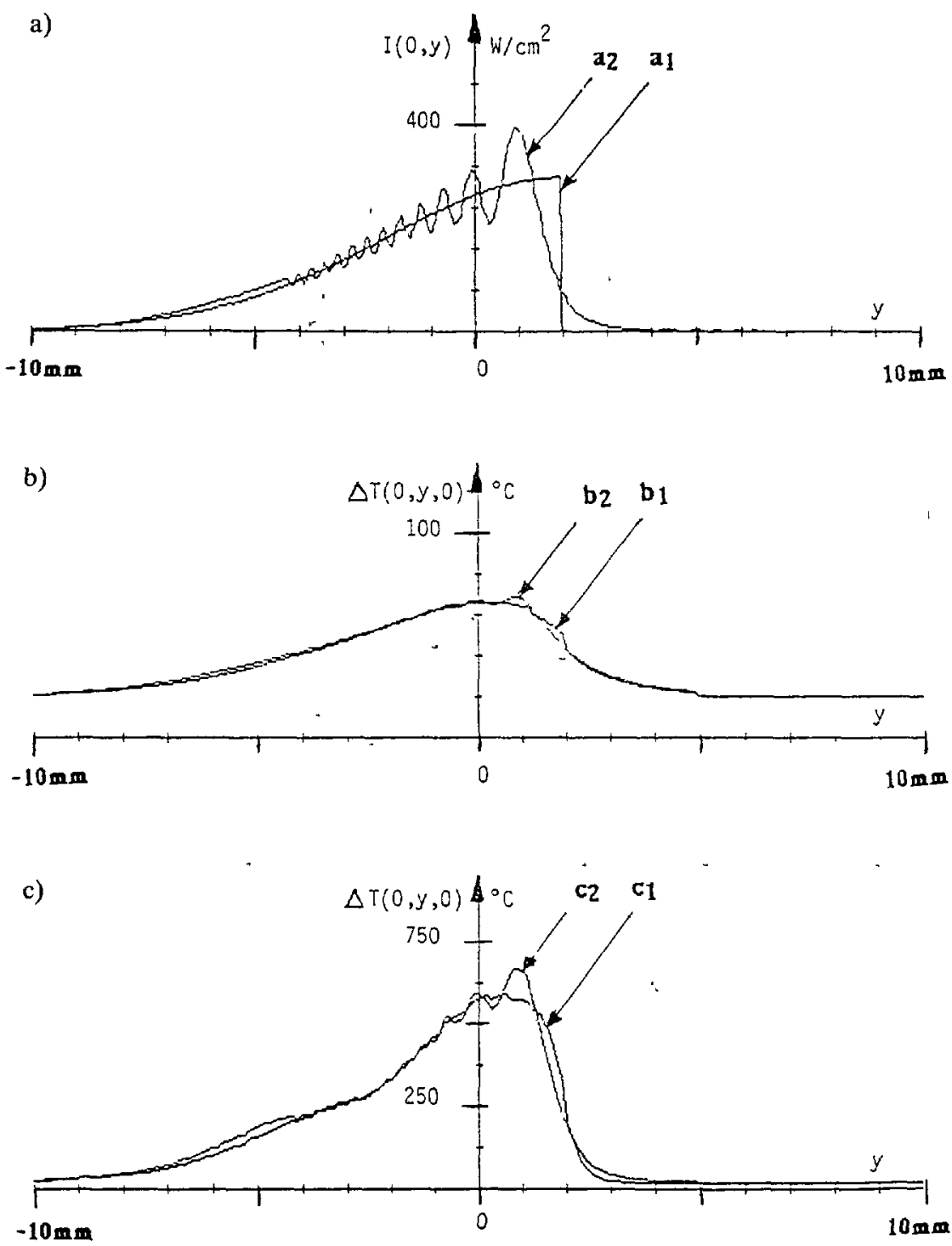

Fig. 5. - Comparaison après une irradiation de $0,5 \mathrm{~s}$ entre les champs de température induits en surface de matériaux conducteur (5b) ou isolant (5c) par un demi-faisceau gaussien en tenant compte $\left(5 a_{2}\right)$ ou non $\left(5 a_{1}\right)$ du phénomène de diffraction.

[Comparison of superficial temperature fields induced on conducting ( $5 b$ ) or insulating (5c) material by an half Gaussian beam with $\left(5 \mathrm{a}_{2}\right)$ or without $\left(5 \mathrm{a}_{1}\right)$ diffraction phenomenon.]

sera d'autant plus net que le temps d'interaction sera court, et le matériau sera mauvais conducteur. Nous pouvons d'ailleurs considérer que les impacts des faisceaux sur le papier thermique (Fig. 4) constituent dans une certaine mesure une vérification expérimentale de ce point.

4.2 POSSIBILITÉ D'OBTENTION D'UNE RÉPARTITION DE TEMPÉRATURE HOMOGẼNE PAR RÉGLAGE DU DISPOSITIF OPTIQUE. - En utilisant les deux groupes de résultats numériques 

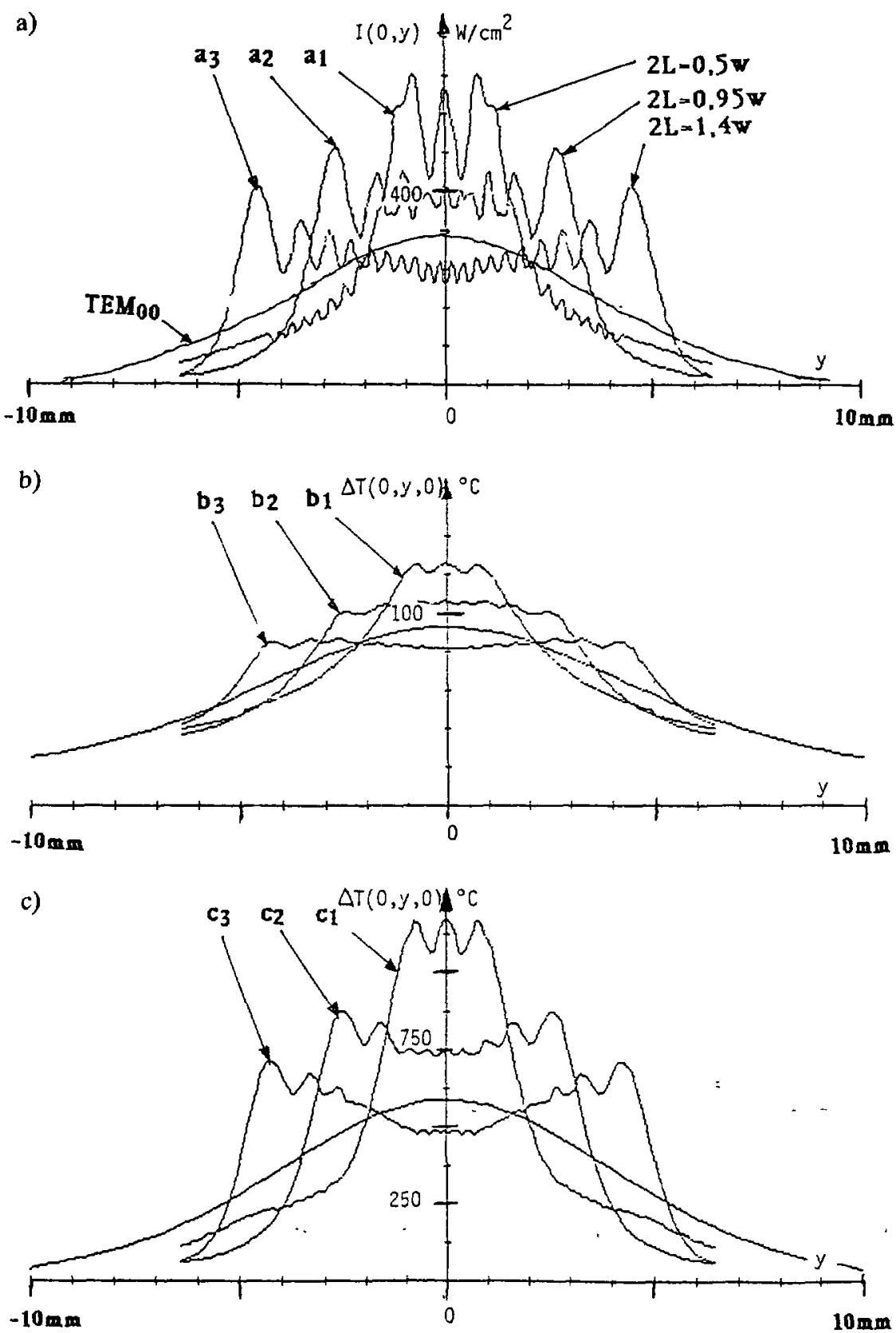

Fig. 6. - Résultats obtenus après recombinaison de deux sous-faisceaux symétriques : a) exemples de répartitions énergétiques pouvant être obtenues pour des largeurs de bandes gaussiennes $(2 L)$ variables entre 0,5 et 1,4 rayon $(w)$ du faisceau primaire; b) exemples de champs de température obtenus sur un matériau conducteur (acier) avec les répartitions présentées et après une irradiation de $0,5 \mathrm{~s} ; \mathrm{c}$ ) champs de température qui seraient obtenus sur un matériau isolant (céramique) avec les mêmes paramètres qu'en $b$ ).

[Obtained results after recombination of two symmetrical half-beams: a) examples of energy distribution which can be observed; b) examples of thermal fields which can be observed on a conducting material with these energy distributions; c) temperature fields which would be able to observe on insulating material with the same energy distributions.] 
précédents (sur l'acier et la céramique), nous avons simulé la répartition de température superficielle pour quelques combinaisons des deux sous-faisceaux. Les résultats sont donnés sur la figure 6 pour trois réglages du dispositif ainsi que pour le faisceau gaussien direct afin de pouvoir juger de l'influence du dispositif. Il est clair que l'on peut obtenir une répartition de température sensiblement homogène par ce dispositif (voir Figs. 6b3, 6c2). On constate aussi que le réglage du dispositif conduisant à cette situation est différent pour les deux matériaux. Une répartition de température homogène nécessite une largeur de «bande gaussienne » $2 L$ égale environ au rayon du faisceau (soit $1 w$ ) au niveau de la surface de la céramique, mais à $1,4 w$ pour l'acier. C'est-à-dire que si l'on souhaite obtenir une répartition de température homogène sur un matériau bon conducteur après des temps d'interaction relativement grands, la forme de la répartition doit ressembler à celle d'une « selle de cheval ». Et on peut

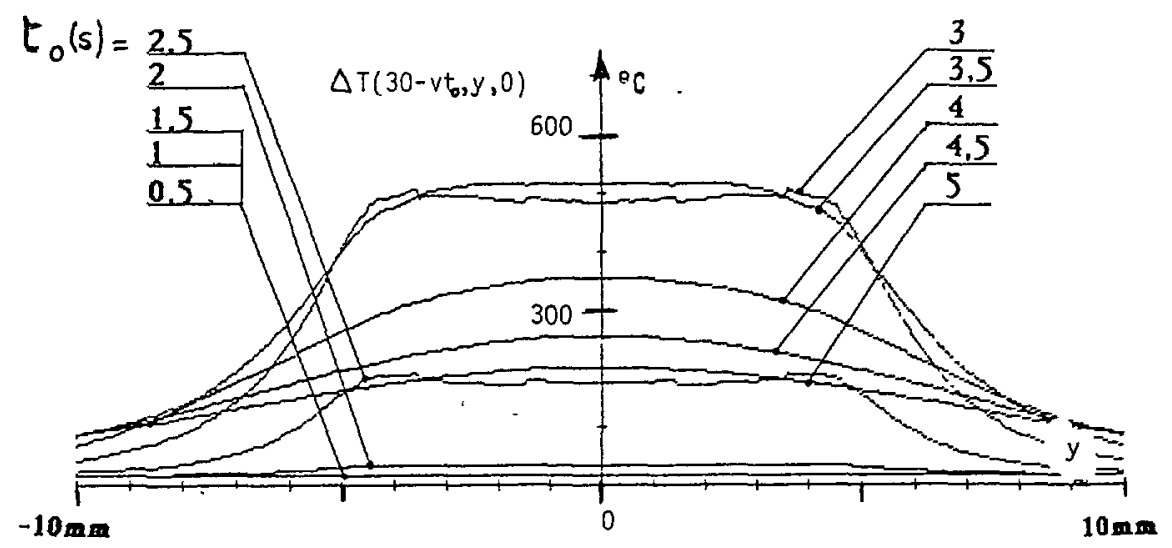

Fig. 7. - «Homogénéisation » de la température superficielle par balayage à $10 \mathrm{~mm} / \mathrm{s}$ de la surface des matériaux avec la répartition en "bande gaussienne" de la figure $6 a-a_{3}$ pour différents instants d'observation $t_{0}$.

[« Homogenization » of superficial temperature with scanning of material surface by a « Gaussian strip » distribution.]

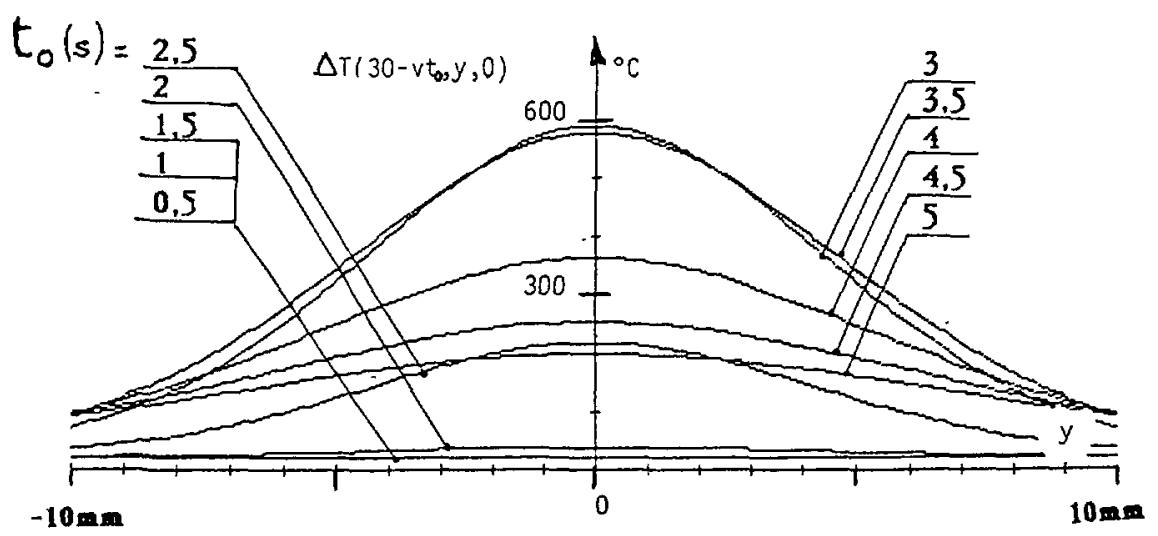

Fig. 8. - Répartitions de température obtenues avec un balayage du faisceau gaussien non transformé par le dispositif optique.

[Temperature distribution obtained by scanning with an « ideal » Gaussian beam.] 
considérer que pour un temps fixe cette forme sera d'autant plus relevée sur les bords que la conductivité thermique du matériau sera grande. Par contre, si l'on désire obtenir une répartition de' température homogène en surface sur des matériaux mauvais conducteurs, il faudra utiliser un dispositif permettant de superposer des images de sous-faisceaux [1,3].

Enfin; pour simuler le traitement par balayage (donc à $v \neq 0$ ), nous présentons, figure 7 , un ensemble de courbes calculées avec les paramètres suivants : matériau : acier ; déplacement à la vitesse $v=10 \mathrm{~mm} / \mathrm{s}$ suivant l'axe $\mathrm{O} x$; réglage du dispositif optique correspondant à la répartition présentée figure $6 \mathrm{a} 3$ avec une puissance absorbée $P_{0}$ de $2000 \mathrm{~W}$. Les résultats présentés sont sensés représenter l'évolution de la température selon une ligne d'observation fixe, parallèle à la direction $y$, située à $30 \mathrm{~mm}$ à l'avant de l'axe du faisceau à l'instant $t_{0}=0$ (córrespondant au début de l'éclairement $\mathrm{du}$ plan d'interaction). Les profils de température présentés correspondent donc aux profils pouvant être successivement observés aux instants $t_{0}$ variables de 0,5 à $5 \mathrm{~s}$ lorsque le faisceau se rapproche puis dépasse la ligne d'observation. Il est clair que l'homogénéité de la répartition de température varie avec l'instant d'observation mais on constate une nette amélioration de cette homogénéité par rapport au cas du faisceau gaussien direct, transportant la même quantité d'énergie, comme cela ressort de la figure 8 .

\section{Conclusion.}

Les études présentées montrent que pour obtenir une température uniforme à la surface d'une pièce, il n'y a pas une répartition d'énergie idéale mais qu'il existe tout au plus des répartitions d'énergie optimales pour un traitement donné sur un matériau donné. Cela signifie qu'il devrait y avoir autant de dispositifs de transformation de faisceau que de cas rencontrés et souligne l'intérêt de dispositifs du type de $L_{2}$ ' Ils sont en effet 'parfaitement capables de remplir ce rôle, du fait de leur possibilité de faire varier continuement la répartition d'énergie par superposition de sous-faisceaux. Les différents dispositifs que nous avons proposés étant tous fondés sur un principe analogue, c'est-à-dire une décomposition du faisceau primaire gáussien en sous-faisceaux recombinés au niveau de lá zone d'interaction, ils devraient donc tous être susceptibles de s'adapter à une assez grande gamme de conditions.

Il faut, par contre, noter que bien que notre analyse soit fondée sur un. modèle idéal de faisceau, en l'occurrence un faisceau $\mathrm{TEM}_{00}$ idéal, les calculs restent relativement compliqués. Dans la pratique. la prise en compte des conditions expérimentales réelles conduira à des calculs encore plus complexes interdisant en particulier tous calculs en temps réel nécessaires si l'on veut modifier le réglage du dispositif optique pour contrôler l'effet thermique induit par l'intermédiaire d'une caméra I.R. En conséquence, nous cherchons à mettre au point des méthodes d'approximation [7] permettant l'évaluation beaucoup plus rapide des champs de température et permettant donc d'espérer la modification des caractéristiques de la répartition d'énergie durant l'opération de traitement thermique par laser.

\section{Annexe A : Interférences induites par deux ondes cylindriques.}

D'après la référence [7], nous pouvons considérer pour le dispositif $L_{2}$, que les interférences sont induites par deux ondes cylindriques centrées sur les deux lignes-focales symétriques des deux miroirs cylindriques. Dans la figure Al, nous donnons le chemin optique pour une de ces ondes. Il est clair que la longueur du chemin optique entre le plan, d'observation $x \mathrm{O} y$ et la ligne-focale peut s'exprimer par :

$$
\begin{aligned}
\ell & =\sqrt{\left[(d-f) \cos \theta+z_{\mathrm{r}}\right]^{2}+[(d-f) \sin \theta-y]^{2}} \\
& =\sqrt{(d-f)^{2}+2(d-f)\left(z_{\mathrm{r}} \cos \theta-y \sin \theta\right)+z_{\mathrm{r}}^{2}+y^{2}} .
\end{aligned}
$$




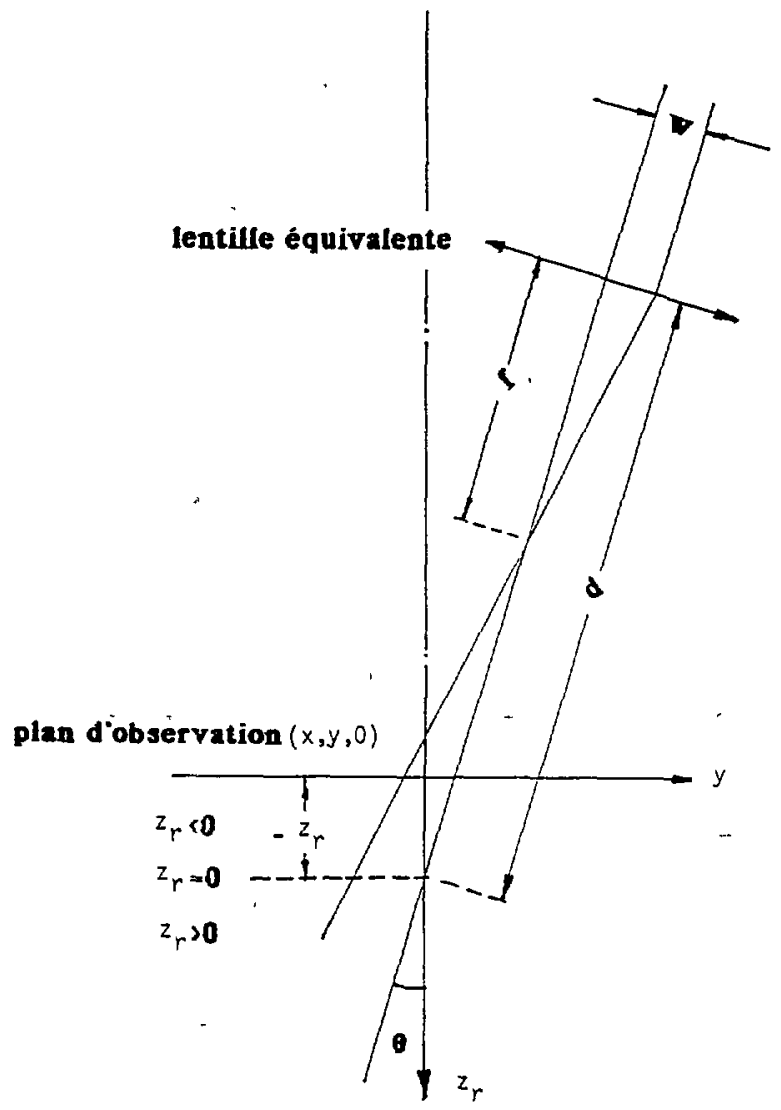

Fig. A.1. - Schéma présentant les chemins optiques du dispositif étudié.

[Optic paths for the studied device.]

Cependant, dans la pratique, on a toujours $|y| \ll(d-f)$ et $\left|z_{\mathrm{r}}\right| \ll(d-f)$, donc on peut négliger les deux derniers termes dans l'expression (1.A) et l'approximer par:

$$
\ell=(d-f) \sqrt{1+\frac{2\left(z_{\mathrm{r}} \cos \theta-y \sin \theta\right)}{d-f}} \approx(d-f)+\left(z_{\mathrm{r}} \cos \theta-y \sin \theta\right) .
$$

Dans ce cas, en négligeant le terme constant, le facteur de phase peut être exprimé par :

$$
\exp \left(j \frac{2 \pi}{\lambda} \dot{\ell}\right)^{\prime}=\exp \left[j \frac{2 \pi}{\lambda}\left(z_{\mathrm{r}} \cos \theta-y \sin \theta\right)\right]
$$

avec $\lambda$, longueur d'onde.

Par conséquent, en utilisant le système de coordonnées repérées dans la figure $\mathrm{A} 1$, nous pouvons exprimer l'amplitude du champ complexe par:

$$
\begin{array}{r}
U(x, y, t)=\frac{1}{\sqrt{M_{y}}} \exp \left[-\frac{(x-v t)^{2}}{w^{2}}\right] \exp \left[-\frac{\left(y \cos \theta+z_{\mathrm{r}} \sin \theta\right)^{2}}{M_{y}^{2} w^{2}}\right] \times \\
\times \exp \left[j \frac{2 \pi}{\lambda}\left(z_{\mathrm{r}} \cos \theta-y \sin \theta\right)\right]
\end{array}
$$


avec

$$
M_{y}=\left|1-\frac{d+z_{\mathrm{r}} \cos \theta-y \sin \theta}{f}\right|
$$

pour

$$
y<-z_{\mathrm{r}} \operatorname{tg} \theta
$$

Et, par raison de symétrie géométrique, on peut trouver la répartition d'énergie sur l'ensemble du plan d'observation:

$$
\begin{aligned}
& E(x, y, t)= \\
& \quad=[U(x, y, t)+U(x,-y, t)] \cdot\left[U^{*}(x, y, t)+U^{*}(x,-y, t)\right] \\
& \quad=|U(x, y, t)|^{2}+|U(x,-y, t)|^{2}+U(x, y, t) U^{*}(x,-y, t)+U^{*}(x, y, t) U(x,-y, t) .
\end{aligned}
$$

Dans cette expression, les deux premiers termes correspondent à la somme des répartitions d'énergie des deux ondes cylindriques, et les autres décrivent les perturbations induites par le phénomène d'interférence.

D'après l'expression (3.A) on obtient donc:

$$
\begin{aligned}
& |U(x, y, t)|^{2}=\frac{1}{M_{y}} \exp \left[-\frac{2(x-v t)^{2}}{w^{2}}\right] \exp \left[-\frac{2\left(y \cos \theta+z_{\mathrm{r}} \sin \theta\right)^{2}}{M_{y}^{2} w^{2}}\right] \quad\left(y \leqslant-z_{\mathrm{r}} \operatorname{tg} \theta\right) \\
& |U(x,-y, t)|^{2}=\frac{1}{M_{y}} \exp \left[-\frac{2(x-v t)^{2}}{w^{2}}\right] \exp \left[-\frac{2\left(y \cos \theta-z_{\mathrm{r}} \sin \theta\right)^{2}}{M_{y}^{2} w^{2}}\right] \quad\left(y \geqslant z_{\mathrm{r}} \operatorname{tg} \theta\right)
\end{aligned}
$$

et

$$
\begin{aligned}
& U(x, y, t) U^{*}(x,-y, t)+U^{*}(x, y, t) U(x,-y, t)= \\
& =\operatorname{rect}\left(\frac{y}{-2 z_{\mathrm{r}} \operatorname{tg} \theta}\right) \frac{2}{M_{y}} \exp \left[-2 \frac{(x-v t)^{2}}{w^{2}}\right] \exp \left[-2 \frac{y^{2} \cos ^{2} \theta+z_{\mathrm{r}}^{2} \sin ^{2} \theta}{M_{y}^{2} w^{2}}\right] \cos \left(\frac{2 \pi}{F_{\mathrm{i}}} y\right) \\
& \text { avec } \\
& \qquad F_{\mathrm{i}}=\frac{\lambda}{2 \sin \theta} .
\end{aligned}
$$

Expressions qui permettent de remonter aux termes $H_{\mathrm{g}}(y)$ et $H_{1}(y)$ décrivant la répartition d'énergie dans ces hypothèses particulières.

\section{Bibliographie -}

[1] Li J. C., Merlin J., Perez J., Rev. Phys. Appl. 21 (1986) 425-433.

[2] Li Junchang, J. Opt. 18 (1987) n 2.

[3] Li Junchang, Merlin J., Manderscheid T., Corbet A., Rev. Phys. Appl. 24 (1989) 1111-1118.

[4] Merlin J., Li J. C., Oliveira C., J. Opt. 21 (1990) 51-61.

[5] VANnes A. B., Laser et Industries de Transformation, Tec et Doc Lavoisier (1986).

[6] Li Junchang, Chinese J. Laser, 15 (1988) No. 11.

[7] Merlin J., Oliveira C., Li J. C., Proceeding of the 2nd IHTF seminar « Surface Engineering with High Energy Beam" (Lisbonne, Sept. 89). 